\title{
The Liquid Crystals of Sodium Salt of Poly(glutamic acid) in Aqueous Solution
}

\author{
Eisaku IIzuka, Yoshiyuki Kondo, and Yoshiro UKaI \\ Institute of High Polymer Research, Faculty of Textile Science and \\ Technology, Shinshu University, Ueda 386, Japan.
}

(Received October 22, 1976)

\begin{abstract}
Liquid crystal structures which are a collection of molecular clusters have been found in concentrated solutions of sodium salt of poly(glutamic acid) by means of polarizing micrograph, scattering of laser light, infrared absorption, X-ray diffraction, circular dichroism, and some other physical methods. This polymer undergoes a coil-helix transition at a certain polymer concentration that depends on the temperature, even at slightly alkaline pHs, and eventually becomes liquid crystalline. With the addition of $50-\%(\mathrm{v} / \mathrm{v})$ dioxane, helical polymers are separated as coacervate and show similar structures.
\end{abstract}

KEY WORDS Poly(glutamic acid) / Liquid Crystal / Coil-Helix Transition /

From ample experimental evidence, one of the authors (E. I. $)^{1}$ has proposed the idea that liquid crystals of the molecular cluster type are formed in concentrated solutions of polypeptides such as poly $(\gamma$-benzyl L-glutamate) (PBLG) and poly( $\gamma$-ethyl L-glutamate) (PELG). The solvents used in the experiments were such organic solvents as dibromomethane, dichloromethane chloride, chloroform, and dioxane. The liquid crystals of living systems are naturally found in aqueous medium and the study of liquid crystals in such a medium is expected to supply more useful information concerning the structure and function of living systems. The purpose of this report is to present some new findings on the concentrated aqueous solutions of the $\mathrm{L}$ - and Disomers of sodium salt of poly(glutamic acid) (PGA).

\section{EXPERIMENTAL}

The L- and D-isomers of sodium salt of PGA (PLGA-Na and PDGA-Na) used were of mean degrees of polymerization (DPs) from 100 to 1200 , and were donated by Dr. S. Mori of the Central Research Laboratory, Ajinomoto Co., Ltd. PLGA-Na, PDGA-Na, or equal proportions of PLGA-Na and PDGA-Na were dissolved in deionized water, and dialyzed against deionized water in a cellophane tubing overnight. Each sample was then concentrated in a constant stream of air, (mixed with appropriate dioxane-water mixtures to make up $50-\%(\mathrm{v} / \mathrm{v})$ dioxane concentration in certain cases) and introduced into a $1-\mathrm{mm}$ quartz cell with a quartz spacer to obtain the desired path lengths near $0.1 \mathrm{~mm}$. The apparent $\mathrm{pH}$ of the concentrated solutions $(10-50 \%(\mathrm{w} / \mathrm{v}))$ was about 8.0.

Polarizing micrographs of the concentrated solutions were taken under crossed polaroids to increase contour, and the low-angle light scattering was measured using a $6328 \mathrm{~A}$ light from a $2-\mathrm{mW} \mathrm{He}-\mathrm{Ne}$ gas laser. Circular dichroism (CD) measurements were carried out with a Jasco ORD/UV-5 spectropolarimeter; the optical cell was mounted on a cell holder that can be rotated in a plane perpendicular to the incident beam. Details of the CD measurements have been described elsewhere. ${ }^{2}$ The solution dissolved in $\mathrm{D}_{2} \mathrm{O}$ was sandwiched between two polyethylene films and measurements of the infrared absorption were made with a Jasco DS301 spectrophotometer. In these measurements, the temperature of the solutions was regulated 
at $24^{\circ} \mathrm{C}$ in a cuvette by circulating water, unless stated otherwise. Wide-angle X-ray diffraction patterns were photographed in a glass capillary tube of diameter $1 \mathrm{~mm}$ with a Rigaku Denki Geigerflex $\left(35 \mathrm{kV}, 25 \mathrm{~mA}, \mathrm{Cu}-\mathrm{K}_{\alpha}\right.$ line), and the steady-state viscosity was also measured with a cone-and-plate viscometer manufactured by Iwamoto Seisakusho Co., Ltd. These measurements were carried out at room temperature $\left(22 \pm 1^{\circ} \mathrm{C}\right)$. The solutions were dried completely to determine the polymer concentration from their weights only very roughly.

\section{RESULTS AND DISCUSSION}

Sodium Salt of Poly(glutamic acid) in Water

The solution of PLGA-Na or of PDGA-Na becomes slightly turbid at a certain polymer concentration and eventually becomes birefringent. This critical concentration has a fixed value of $43.8 \%(\mathrm{w} / \mathrm{v})$ at $24^{\circ} \mathrm{C}$ for the polymers whose DPs are more than 250 (Figure 1). The polymer molecules are initially in the coiled conformation at this $\mathrm{pH}^{3}$ and the appearance of the birefringent phase suggests that the polymer molecules undergo a transition to some ordered conformation; when the birefringent solution is heated up, it becomes isotropic at a certain temperature that again depends on the polymer concentration (Figure 2).

In $\mathrm{D}_{2} \mathrm{O}$, the infrared absorption spectrum of the birefringent solution shows the amide I band

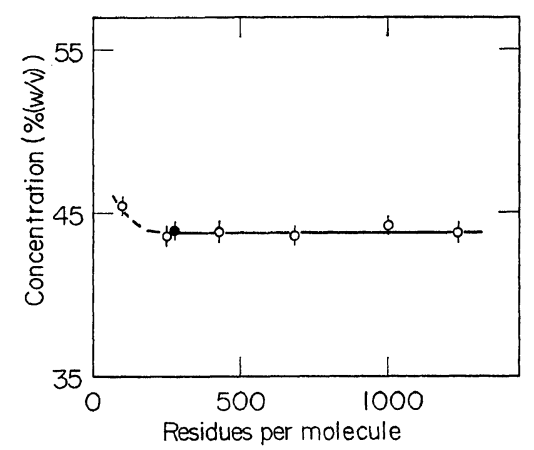

Figure 1. Degree of the polymerization dependence of the critical concentration above which the solution becomes (completely) birefringent: $O$, PLGA-Na in $\mathrm{H}_{2} \mathrm{O}$; $, 1: 1$ PLGA-Na(DP 300)PDGA-Na(DP 250) in $\mathrm{H}_{2} \mathrm{O}$; measured at $24^{\circ} \mathrm{C}$. at around $1650 \mathrm{~cm}^{-1}$ and the amide II band at around $1570 \mathrm{~cm}^{-1}$; These bands are shifted somewhat in wave number when compared with those of the isotropic solution (the left panel of Figure 3), corresponding to the occurrence of some conformational transition. (The peptide groups of this polypeptide are not deuterated, unlike in silk fibroin. ${ }^{4}$ ) From this experimental result, it may be concluded that the ordered conformation expected to appear in the birefringent solution is at least not the $\beta$-form, because this solution lacks the absorption maxi-

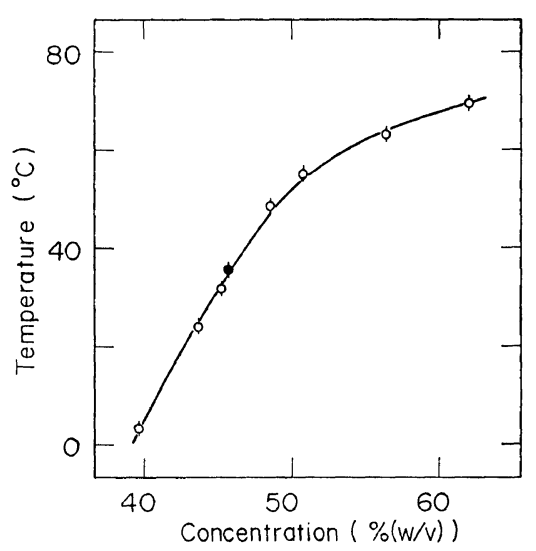

Figure 2. Polymer concentration dependence of the isotropic-birefringent transition temperature: $\bigcirc$, PDGA-Na of DP 680 in $\mathrm{H}_{2} \mathrm{O}$; $, 1: 1$ PLGA$\mathrm{Na}$ (DP 300)-PDGA-Na(DP 250) in $\mathrm{H}_{2} \mathrm{O}$.

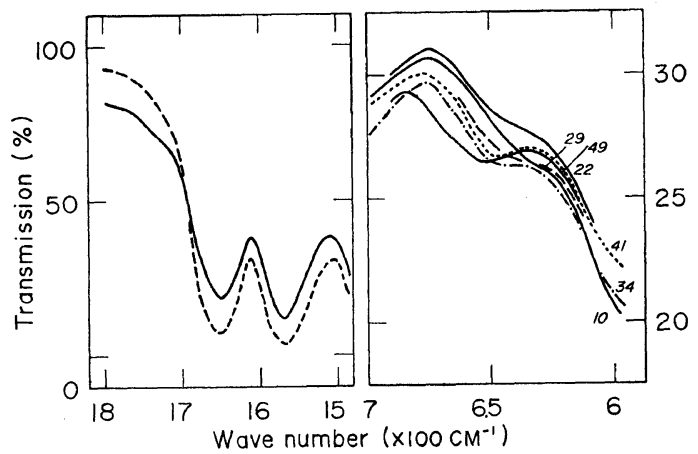

Figure 3. Infrared absorption spectra of PDGA$\mathrm{Na}$ : DP, 680; measured at $24^{\circ} \mathrm{C}$ in $\mathrm{D}_{2} \mathrm{O}$. Lefthand curves: solid line, the birefringent solution; broken line, the isotropic solution. Right-handed curves: numbers on the curves give the temperature of the solution $(45.6 \%(\mathrm{w} / \mathrm{v}))$ in degrees. 
mum centered at $1620-1630 \mathrm{~cm}^{-1}$, and that the ordered conformation is most likely the $\alpha$-helix, because other ordered conformations do not seem probable. $^{5}$ When the birefringent solution $(45.6 \%(\mathrm{w} / \mathrm{v}))$ is heated, its absorption spectrum displays a marked change in the amide- $\mathrm{V}$ band region (the right panel of Figure 3). At $10^{\circ} \mathrm{C}$, there is only one band centered at around $630 \mathrm{~cm}^{-1}$, although obscured by neighboring bands, indicating that the polymer molecules are fully $\alpha$-helical, ${ }^{6}$ with increasing temperature, the intensity of this band diminishes and a new band appears at $650 \mathrm{~cm}^{-1}$ with its intensity increasing with temperature. These indicate the occurrence of the helix-coil transition as has been expected. The drastic change in the feature of the absorption spectrum that occurs around $34^{\circ} \mathrm{C}$ corresponds to the birefringent-isotropic transition. At $29^{\circ} \mathrm{C}$, the change is still not marked; however, at $41^{\circ} \mathrm{C}$, the transition is almost completed. Thus, the transition occurs rather in a narrow temperature range, which was confirmed by the observation under crossed polaroids. When the solution was cooled down, it became birefringent again in exactly the same temperature range in which it had become isotropic.

In dilute aqueous solution of $\mathrm{PGA}-\mathrm{Na}$, its carboxyl groups are completely ionized at $\mathrm{pH}$ 8.0 and the polymer chains are well extended. ${ }^{3}$ The contraction of the polymer chains occurs continuously with increasing salt concentration even above $0.2-1 \mathrm{~mol}$, that should be enough to suppress the electrostatic repulsion. ${ }^{7,8}$ This salt effect has been interpreted as a consequence of very general effects of the various ions on the structure of the solvent, which in turn modify solvent-polymer interactions involved in the stability of the native structure. ${ }^{8}$ The increase of polymer concentration would cause the decrease of thermodynamic activity of the solvent water in a similar manner and invite the coil-helix transition. Quite a similar molecular mechanism must be operative as in the concentration-induced coil-helix transition of PBLG in dichloroacetic acid (DCA) found by Frenkel', et al. ${ }^{9}$

The measured $\mathrm{CD}$ of the birefringent solution shows a dependence of the macroscopic rotation angle of the optical cell with respect to the

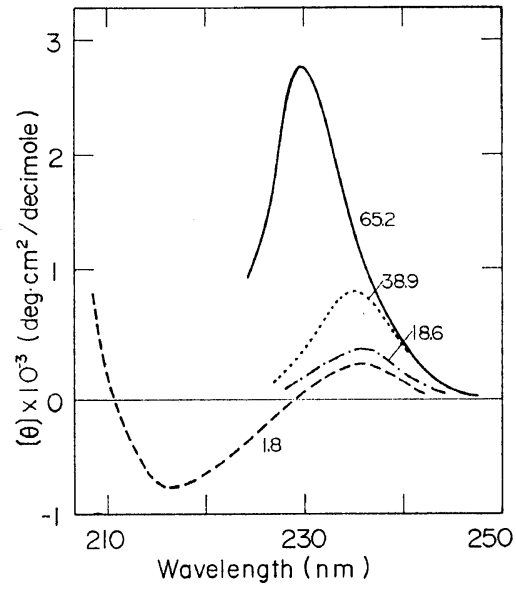

Figure 4. Circular dichroism spectra of the solutions of PDGA-Na: DP, 250; measured at $27^{\circ} \mathrm{C}$ in $\mathrm{H}_{2} \mathrm{O}$. The numbers on the curves give the polymer concentration in $\%(w / v)$.

vertical position because of both linear and circular birefringence and linear and circular dichroism of the (oriented) specimen. If the specimen is not highly oriented and/or is thin enough, the real $C D$ curve is given as the average of the apparent CD curves. ${ }^{10}$ The real CD curve of the birefringent solution of PDGA$\mathrm{Na}$ differs from that of the isotropic solution, reflecting the coil-helix transition of the polymer molecule (Figure 4). In the birefringent solution, there is only one peak at $230 \mathrm{~nm}$, whereas the $\alpha$-helices (D-isomer in dilute solution) are expected to show double peaks at 222 and $206 \mathrm{~nm}^{3}$ Furthermore, the intensity of the CD is far smaller than expected. These results would suggest the presence of scattering and reflection of the incident beam from the solution, and in turn the formation of some long range organization in solution.

The birefringent solution is very viscous, and is easily oriented by stresses which shear the solution during the insertion of the spacer into the optical cell. Therefore, the feature of the polarizing micrograph of the birefringent solution differs from solution to solution (Figure $5 a, b)$. The $V_{h}$ pattern of the birefringent solution is circular, or of the $\pm 45^{\circ}$ type (Figure 6), suggesting the presence of birefringent assemblies (molecular clusters) of the polymer 


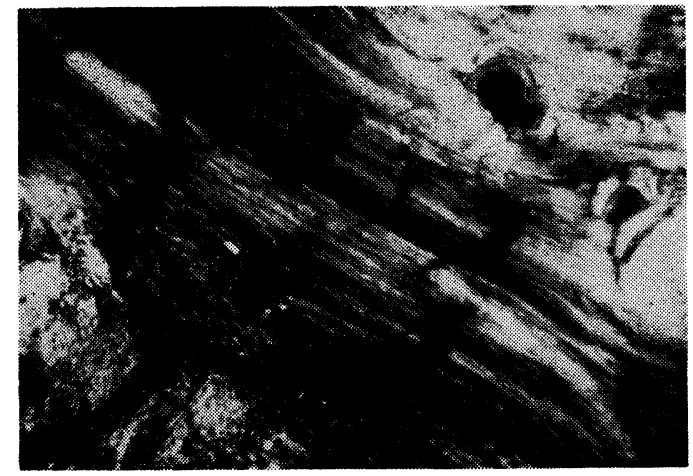

(a)

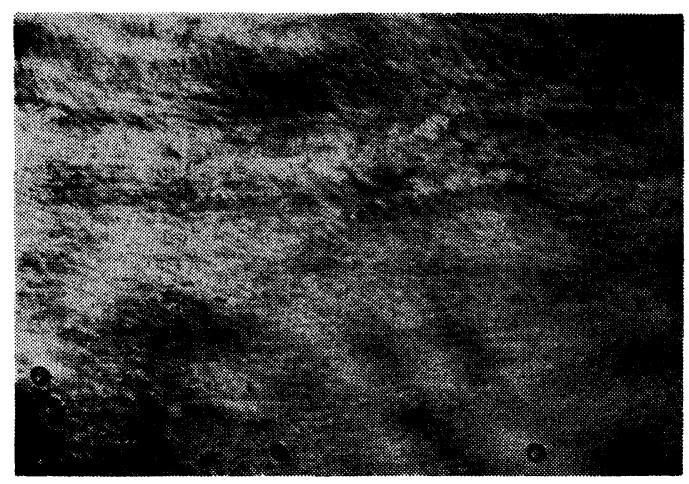

(b)

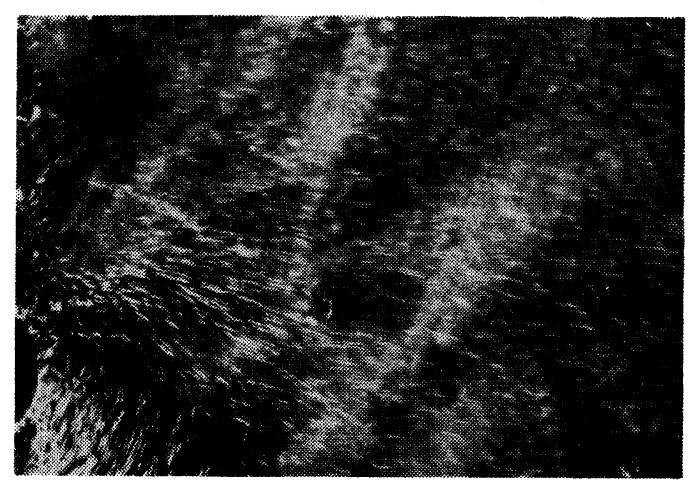

(c)

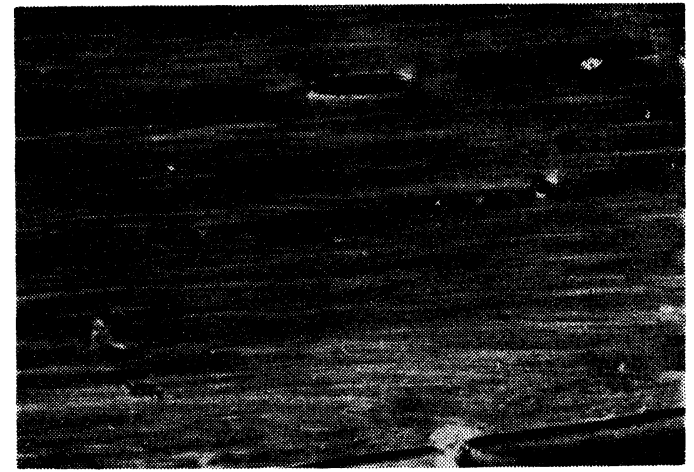

(d)

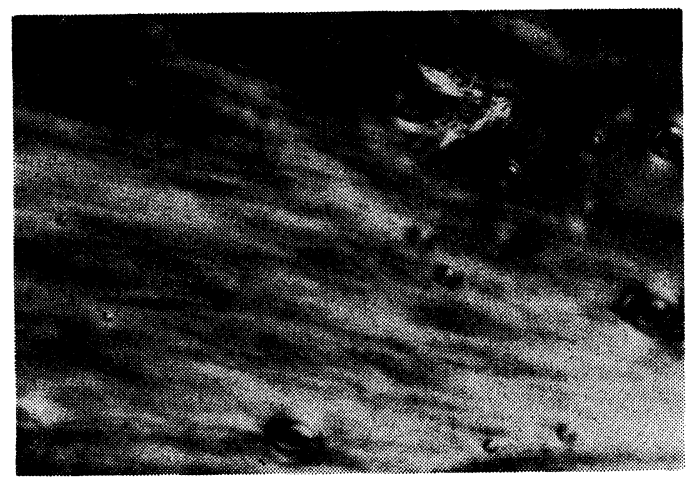

(e)

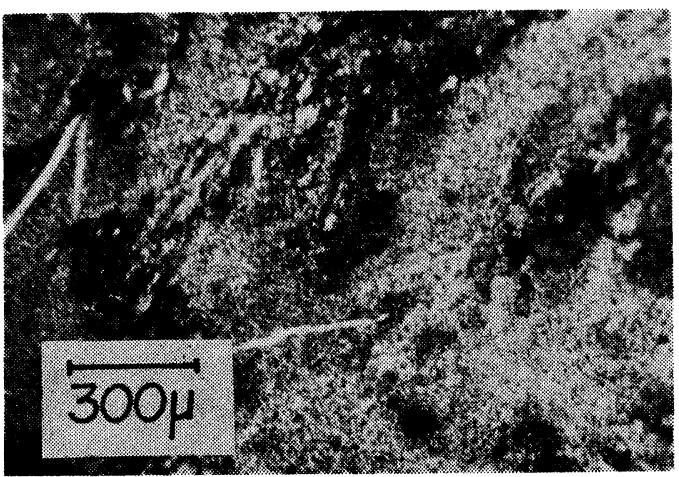

(f)

Figure 5. Polarizing micrographs of the birefringent solutions of PLGA-Na: DP, 300; measured at $24^{\circ} \mathrm{C}$. (a)-(d): dissolved in $\mathrm{H}_{2} \mathrm{O}$ to make up about $50 \%(\mathrm{w} / \mathrm{v})$ solutions; (a), (b) typical patterns; (c) cooled down from the isotropic state at $60^{\circ} \mathrm{C}$ to make the solution birefringent again; (d) sheared (the stripes appear in the direction of shear stresses); (e)-(f): the coacervate of PLGA-Na from a $50 \%(\mathrm{v} / \mathrm{v})$ dioxane solution; (f) cooled down from the isotropic state at $30^{\circ} \mathrm{C}$ to make the specimen isotropic again. 

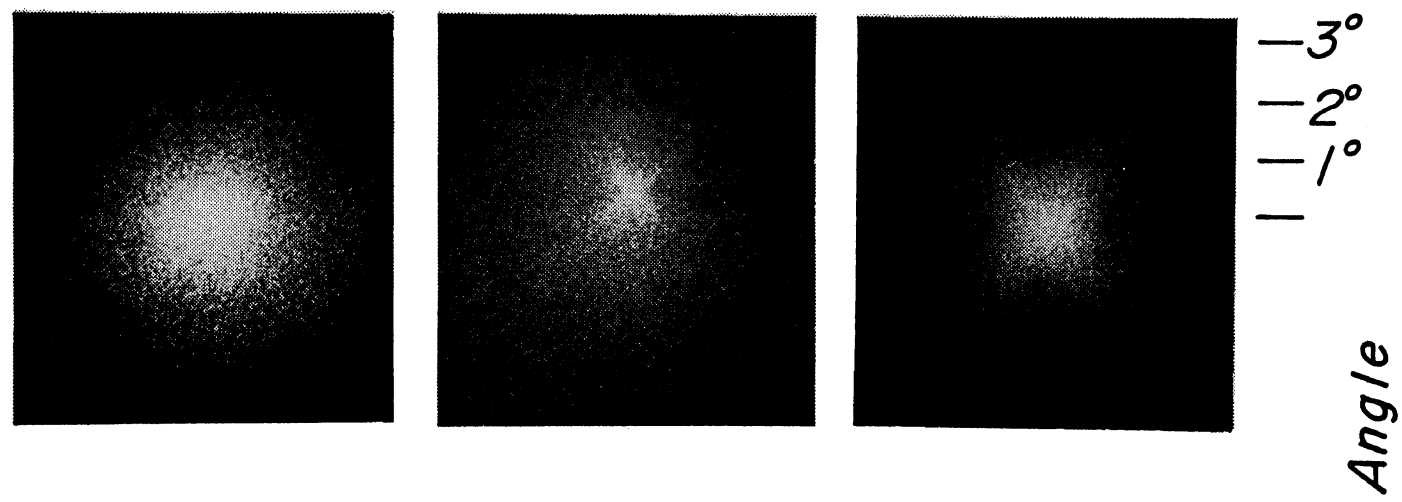

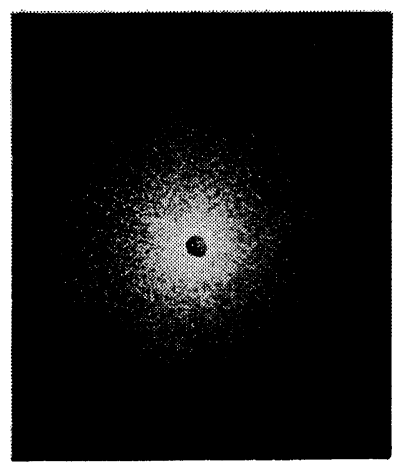

a

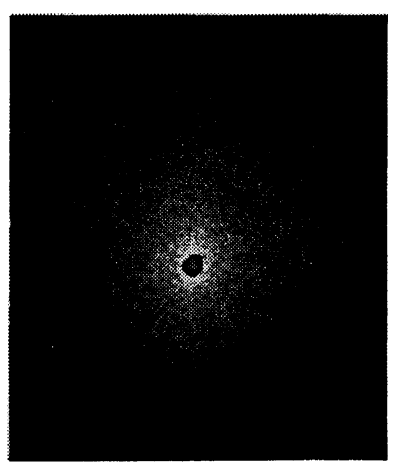

$b$

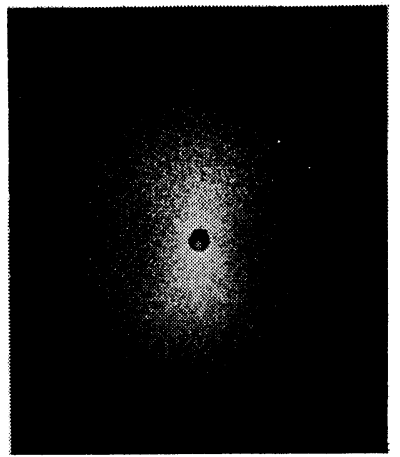

C

Figure 6. Small-angle laser-light-scattering patterns of the birefringent solutions of PLGA$\mathrm{Na}$ : DP, 300; measured at $24^{\circ} \mathrm{C}$ in $\mathrm{H}_{2} \mathrm{O}$. Polymer concentration, $45 \%(\mathrm{w} / \mathrm{v})$. Upper, the $V_{h}$ patterns; the lower, the $H_{h}$ patterns. The pattern (c) corresponds to the micrograph in Figure 5c.

molecules of the order of size comparable to the wavelength of light used. The cross pattern ( $\pm 45^{\circ}$ type pattern) also suggests that the solution is a collection of the rod-like molecular clusters having maximum polarizability directions parallel or perpendicular to their (long) rod axes. ${ }^{11,12}$ As will be mentioned later, the circular pattern turns very easily to the cross pattern under shear stresses, suggesting that the molecular clusters become rod-like. This will explain the reason why the birefringent solution gives the circular pattern in some cases and the cross pattern in other cases. When the birefringent state is regained upon cooling down from the isotropic state at high temperatures, needle-like particles $50-150 \mu$ long appear in the whole range of observation under the microscopic stage (Figure 5c) and the $V_{h}$ pattern is always of the $\pm 45^{\circ}$ type, more distinctly than in the initial state (Figure $6 \mathrm{c}$ ). The $\mathrm{H}_{\mathrm{h}}$ patterns do not give much information concerning the liquid crystal structure in this measurement.

The X-ray diffraction photograph of the isotropic solution shows an amorphous pattern because there is no ordered structure in solution, whereas the birefringent solution shows several Debye-Scherrer rings that correspond most likely to a hexagonal structure of the $\alpha$-helical molecules in the molecular clusters (Figure 7). The $a$-dimension (chain interval) of the corresponding unit cell decreases with polymer concentration (Table I) as in the case of the spacing of the liquid crystals of PBLG. ${ }^{13}$ The $a$-dimensions obtained are close to or larger than those obtained for single crystals of various salts of PGA, in which the dimensions range 

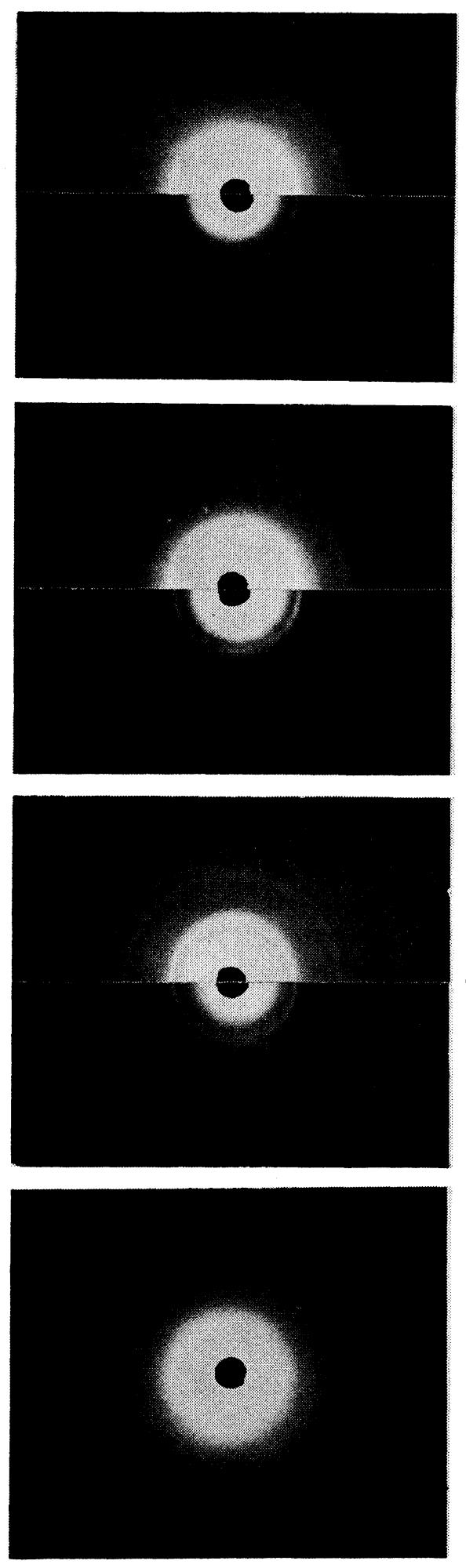

from 12 to $17 \mathrm{~A}$ depending on the environment where the crystals were produced. ${ }^{14}$ When the needle-like particles appear in solution, the $a$ dimension decreases (e.g., from 18.3 A to $17.7 \mathrm{~A}$ at $44 \%(\mathrm{w} / \mathrm{v}))$ and the corresponding DebyeScherrer ring becomes clearly narrow (Figure $7 \mathrm{c})$, suggesting the formation of crystals. It has not been possible to detect the transition temperature below which only the (solid) crystals can exist and thus to complete the phase diagram (Figure 2).

These experimental results may lead to the conclusion that the liquid crystals of the molecular cluster type are formed in the concentrated solutions of PLGA-Na or of PDGA$\mathrm{Na}$. The molecular clusters that form the liquid crystals easily become rod-like under shear

Table I. Lattice spacing of the liquid crystals of sodium salt of poly(glutamic acid)

\begin{tabular}{cc}
\hline $\begin{array}{c}\text { Polymer concentration, } \\
\%(\mathrm{w} / \mathrm{v})\end{array}$ & $a, \mathrm{~A}$ \\
\hline 44 & 18.3 \\
44 & $17.7^{\mathrm{a}}$ \\
43.6 & 18.4 \\
48.6 & 17.9 \\
54.0 & 16.5 \\
59.6 & 16.4 \\
\hline a Cooled down from the isotropic state at $60^{\circ} \mathrm{C}$ to \\
make the solution birefringent again.
\end{tabular}

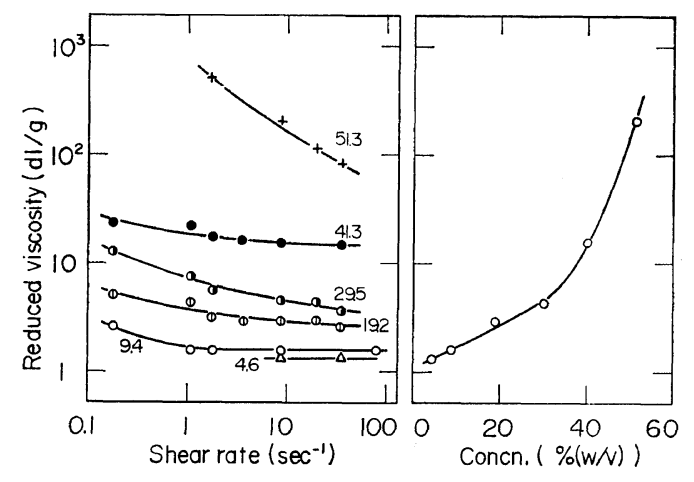

Figure 8. Reduced viscosity vs. shear rate and reduced viscosity $v s$. polymer concentration relationships of PDGA-Na: DP, 250; measured at $24^{\circ} \mathrm{C}$ in $\mathrm{H}_{2} \mathrm{O}$. Left panel, the numbers on the lines give the polymer concentration in $\%(w / v)$; right panel, the values obtained at the shear rate of $9.4 \mathrm{sec}^{-1}$ are plotted.

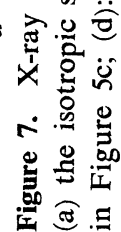



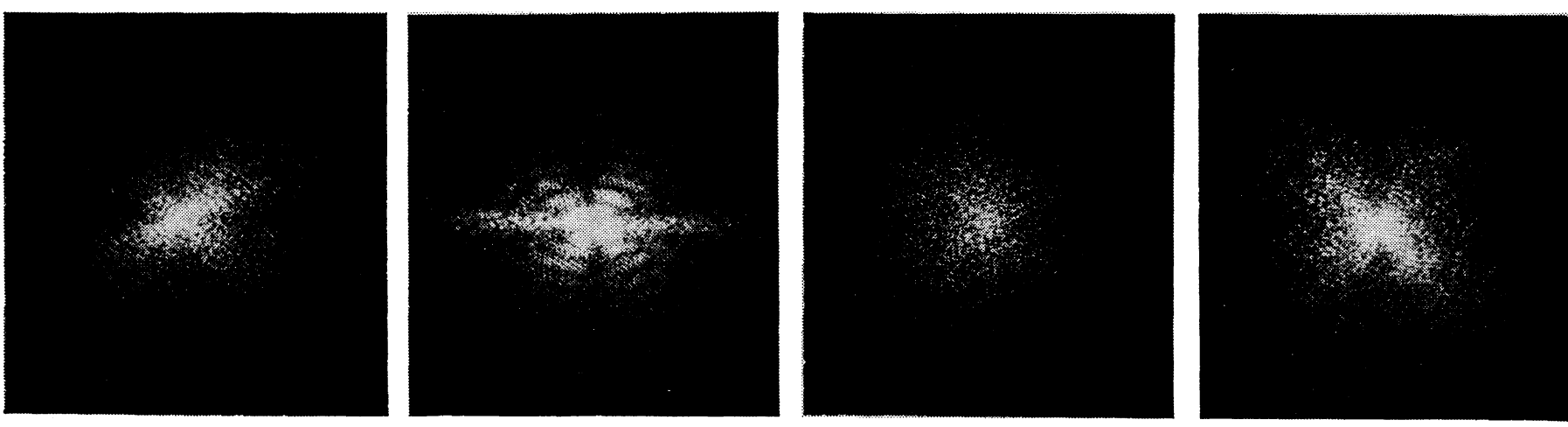

$-3^{0}$
$-2^{0}$
$-1^{0}$

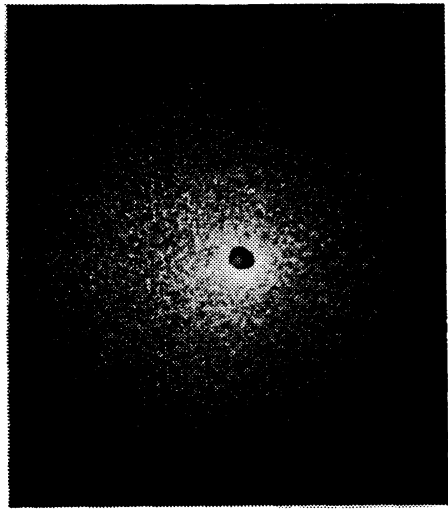

a

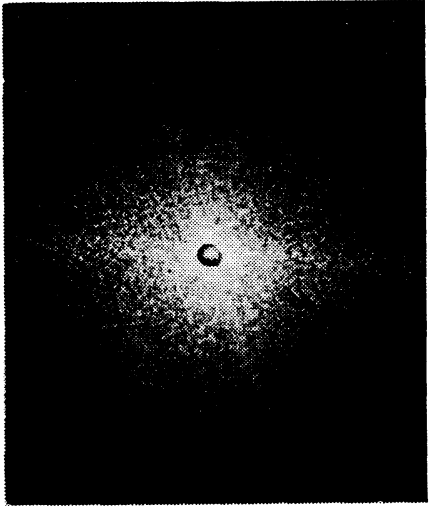

$b$

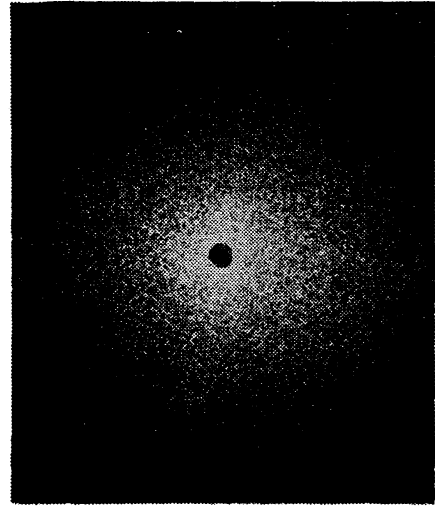

C

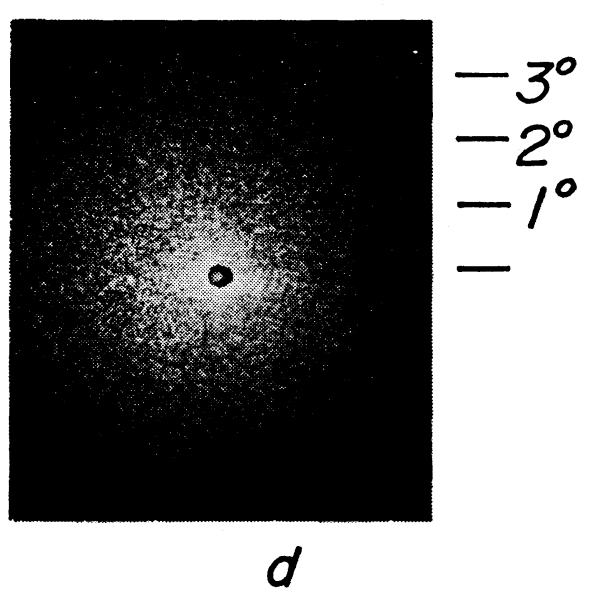

$\frac{0}{8}$

Figure 9. Small-angle laser-light-scattering patterns of the birefringent solutions of PLGA-Na: Upper, the $V_{h}$ patterns and lower, the $H_{h}$ patterns; DP, 300; measured at $24^{\circ} \mathrm{C}$. (a)-(b): dissolved in $\mathrm{H}_{2} \mathrm{O}$, and sheared vertically; (b) sheared longer. (c)-(d): the coacervate of PLGA-Na from a 50\%(v/v) dioxane solution; the patterns (c) and (d) correspond respectively to Figures $5 e$ and $\mathrm{f}$. 
stresses, and these liquid crystals are very similar to those of polypeptides in organic solvents, ${ }^{1,15}$ except that the former is apt to become crystalline when cooled down, especially from the isotropic state realized at a high temperature. In PBLG-DCA preparation, the sequence of isothermic transitions from one ordered state to other takes place immediately after the polymer undergoes an isothermic coil-helix transition at a certain polymer concentration, as the result of restriction of rotation of the $\alpha$-helical polymer molecule ${ }^{9}$. The appearance of the liquid crystals of PGA appears to follow a similar mechanism. These liquid crystals are lyotropic, and have the nature of thermotropic liquid crystals as well. Quite recently, Takahashi and his coworkers $^{16}$ suspected the presence of liquid crystals in a similar system on the basis of their observation under crossed polaroids. Now, their suspicion has turned out to be true.

In PLGA-Na of DP 100, the formation of the liquid crystals occurs at a slightly higher polymer concentration than the critical concentration at which the coil-helix transition is mostly completed (Figure 1). The critical polymer concentration for liquid crystal formation is a function of the axial ratio (and DP) of the rod-like polymer molecule, and increases with decreasing DP. ${ }^{13,17}$ This would explain the above discrepancy.

The steady-state viscosity of the concentrated solutions of PLGA-Na or of PDGA-Na decreases with increasing rate of shear, especially in the liquid crystalline state (the left-hand curves in Figure 8). This decrease in the viscosity would be due to the orientation of the $\alpha$-helical polymer molecules (or rod-like molecular clusters); the destruction of the molecular clusters may be involved when in the liquid crystalline state. ${ }^{18}$ The steady-state viscosity increases markedly upon the formation of liquid crystals (the right-hand curve in Figure 8), and this phenomenon contrasts with those seen both in lyotropic liquid crystals of polypeptides in organic solvents ${ }^{18,19}$ and in thermotropic liquid crystals. ${ }^{20}$ The polymer molecules would aggregate to form a three-dimensional structure having some long range organization and produce higher viscosity when the liquid crystal is formed, in which the conformational transition is naturally involved. These liquid crystals of PGA resemble those of deoxygenated sickle-cell hemoglobin ${ }^{21}$ in their viscosity properties, and this viscosity increase may or may not be characteristic of aqueous liquid crystals.

PLGA-Na and PDGA-Na behaved essentially in the same way concerning liquid crystal formation. An equimolar mixture of PLGA-Na and PDGA-Na becomes liquid crystalline at the same polymer concentration as single PLGA-Na does when the temperature is the same (Figures 1 and 2).

Effects of Shear Stresses on the Liquid Crystals of Sodium Salt of Poly(glutamic acid) in Water

When the quartz spacer which was inserted into the optical cell is moved in and out (but only part of the way) to exert shear stresses upon the liquid crystalline solution of PLGA$\mathrm{Na}$ or of PDGA-Na, a striped pattern in which the stripes are in the direction the solution was sheared appears (Figure 5d); excess shearing causes the disappearance of the birefringent phase, showing the destruction of the liquid crystals. When the optical cell is placed so that these stripes are vertical, the upper (and the lower) included angle of the $\pm 45^{\circ}$ type $V_{h}$ pattern (if it is not observed initially, it appears under shear stress) is at first larger than $90^{\circ}$ (Figure 9a). This indicates that the rod-like molecular clusters are (formed and) oriented in the shear direction. ${ }^{12,21}$ The $V_{h}$ pattern then displays a drastic change, probably suggesting the formation of some different three-dimensional structure (Figure 9b); however, it disappears under further exposure to the shear stresses, accompanying the destruction of liquid crystals. The linear birefringence measurements suggest that the $\alpha$-helical molecules are in the shear direction (and in the direction of the long rod axis of the molecular cluster); however, the maximum double refractive index obtained is only $4.6 \times$ $10^{-4}$. This does not necessarily mean that the orientation of (rod-like) molecular clusters is very poor, or rather, means that the ordering of the polymer molecules is very poor in each molecular cluster.

\section{Sodium Salt of Poly(glutamic acid) in 50-\%(v/v) Dioxane}

PGA has been known to become fully $\alpha$ - 
helical at $\mathrm{pHs}$ below 8.0 by the addition of $50 \%-(\mathrm{v} / \mathrm{v})$ dioxane to its solution. ${ }^{8}$ Although this finding had been obtained in dilute solutions, it was considered to apply as well to concentrated solutions and to lead to the finding of liquid crystals.

A very viscous, opaque phase (coacervate) appears at the bottom of a sample tube at polymer concentrations above $1 \%(\mathrm{w} / \mathrm{v})$ at $24^{\circ} \mathrm{C}$, and its content increases with the polymer concentration. This phase is birefringent (Figure $5 e, f)$, and has the polymer concentration of about $44 \%$ (w/v) when the DP is more than 250. The supernatant solution is isotropic, and has the polymer concentration that decreases with the whole polymer concentration, reaching zero at about $20 \%(\mathrm{w} / \mathrm{v})$. When the coacervate is heated up to say $30^{\circ} \mathrm{C}$, it becomes isotropic.

The supernatant solution shows a $C D$ curve typical of the colied conformation, while the coacervate shows a $C D$ curve which is very similar to that of the birefringent solution obtained without dioxane (Figure 10). The intensities of the $\mathrm{CD}$, however, are different because of possible scattering and reflection effects of incident light on the $\mathrm{CD}$, due to some long range organization in solution that differs between the two. The polarizing micrograph of the coacervate is very similar to that of the birefringent solution, and needle-like particles appear upon cooling down from the isotropic

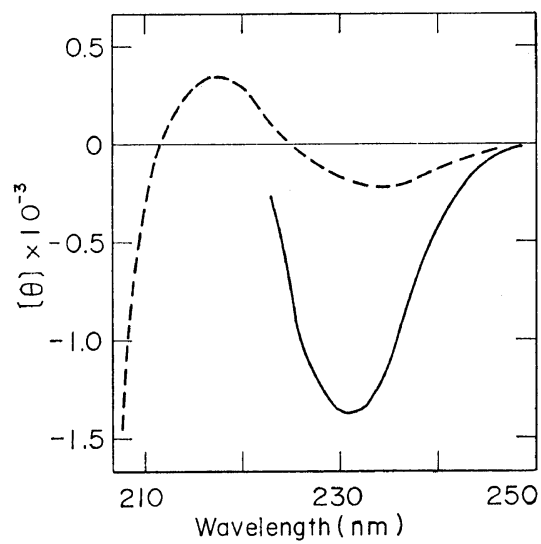

Figure 10. Circular dichroism spectra of the preparation of PLGA-Na-50\%(v/v) dioxane: Measured at $27^{\circ} \mathrm{C}$. Solid line, the coacervate from a $9-\%(\mathrm{w} / \mathrm{v})$ solution; broken line, the supernatant solution. state at higher temperatures as before (Figure $5 e, f)$. The $V_{h}$ pattern is again circular, or of the $\pm 45^{\circ}$ type (Figure 9c, d), and the X-ray diffraction pattern suggests the hexagonal packing of the $\alpha$-helical molecules (Figure 7d). The $a$-dimension of the corresponding unit cell is $18.1 \mathrm{~A}$, which is very close to the value obtained for the birefringent solution having the same polymer concentration $(44 \%(\mathrm{w} / \mathrm{v}))$ (Table I). All these experimental results indicate that the coacervate forms the liquid crystals similar to those found in the concentrated solutions without dioxane.

\section{CONCLUSIONS}

The experimental evidence so far obtained appears to support the following hypotheses concerning concentrated aqueous solutions of sodium salt of poly(glutamic acid):

1. In water having a slightly alkaline $\mathrm{pH}$, this polymer undergoes an isothermal coil-helix transition at a certain polymer concentration that depends on the temperature, but not on the degree of polymerization. Immediately after this transition, the liquid crystals of the molecular cluster type are formed in solution.

2. In liquid crystalline state, the polymer molecules aggregate in the hexagonal array and form assemblies that become rod-like very easily under shear stresses. The interhelical spacing in each assembly decreases with polymer concentration.

3. Under shear stresses, the rod-like assemblies (molecular clusters) are oriented in the shear direction, change their arrangement in solution, but are finally destroyed by too much exposure to the shear stresses.

4. The liquid crystals of this polymer are apt to crystallize (but partly) when being cooled down, especially from the isotropic state realized at high temperatures.

5. Similar liquid crystals are formed also in the coacervate that is separated from the solution of this polymer by the addition of $50-\%(\mathrm{v} / \mathrm{v})$ dioxane.

Acknowledgments. We wish to thank Dr. S. Mori for the supply of polypeptide specimens used. Thanks are also due Miss T. Uehara and 
Miss A. Oka for carrying out parts of the measurements. This work was supported partly by a Grant-in-Aid for Individual Research (058028) awarded to one of us (E. I.) from the Ministry of Education.

\section{REFERENCES}

1. E. Iizuka, "Advances in Polymer Science," Vol. 20, Springer-Verlag, Verlin, 1976, pp 79107.

2. E. Iizuka and J. T. Yang, Mol. Cryst. Liq. Cryst., 29, 27 (1974).

3. G. Holzwarth and P. Doty, J. Am. Chem. Soc., 87, 218 (1965).

4. E. lizuka and J. T. Yang, Proc. Nat. Acad. Sci. U.S., 55, 1175 (1966).

5. T. Miyazawa and E. R. Blout, J. Am. Chem. Soc., 83, 712 (1961).

6. T. Miyazawa, Y. Masuda, and K. Fukushima, J. Polym. Sci., 62, S62 (1962).

7. P. H. von Hippel and K. Y. Wong, Science, 145, 577 (1964).

8. E. lizuka and J. T. Yang, Biochemistry, 4, 1249 (1965).
9. S. Ya Frenkel', L. G. Shaltyko, and G. K. Elyashevich, J. Polym. Sci., Part C, No. 30, 47 (1970).

10. M. J. B. Tunis-Schneider and M. F. Maestre, J. Mol. Biol., 52, 521 (1970).

11. M. B. Rhodes and R. B. Stein, J. Polym. Sci., Part A-2, 7, 1539 (1969).

12. E. Iizuka, T. Keira, and A. Wada, Mol. Cryst. Liq. Cryst., 23, 13 (1973).

13. C. Robinson, J. C. Ward, and R. S. Beevers, Disc. Faraday Soc., 25, 29 (1958).

14. H. D. Keith, G. Giannoni, and F. J. Padden, Jr., Biopolymers, 7, 775 (1969).

15. E. Iizuka, Biochim. Biophys. Acta, 243, 1 (1971).

16. J. Takahashi, S. Shiraishi, and S. Oya, Polymer Reprints, Japan, 24, 883 (1975) (in Japanese).

17. P J. Flory, Proc. Roy. Soc. (London), A234, 73 (1956).

18. E. Iizuka, Mol. Cryst. Liq. Cryst., 25, 287 (1974).

19. J. Hermans, Jr., J. Colloid Sci., 17, 638 (1962).

20. G. W. Gray, "Molecular Structure and the Properties of Liquid Crystals," Academic Press, London, 1962, Chapter 6.

21. E. Iizuka, submitted for publication in Proceedings of the VIth International Liquid Crystal Conference, Kent, Ohio, USA, Aug., 1976. 